\title{
Local and Nonlocal Comparative \\ Static Analysis of Economic Systems*
}

\author{
Robert Kalaba, Leigh Tesfatsion, $\nmid$ and Jone-Lin Wang \\ University of Southern California \\ Los Angeles, California 90007
}

\begin{abstract}
A complete differentialequation system has been developed for the local and nonlocal comparative static analysis of general parametrized economic systems $\Psi(x, \alpha)=0$. The differential equations allow both qualitative and quantitative sensitivity studies at parameter points or over parameter intervals. A FORTRAN program COMSTAT is now available for the numerical integration of the differential equations. The only input requirements are $\Psi(\cdot)$ and initial conditions. Initialization is rendered routine by a two-stage application of comsTAT. Derivatives are automatically evaluated via a generalized Wengert method, freeing the user from ever having to form analytical derivatives. COMSTAT is applied to a problem in optimal economic growth to show the sensitivity of the steady-state capital-labor ratio to changes in the time preference parameter.
\end{abstract}

\section{INTRODUCTION}

Comparative static problems in economics typically reduce to determining the response of a vector $x=\left(x_{1}, \ldots, x_{n}\right)$ to changes in a scalar parameter $\alpha$, where $x$ and $\alpha$ satisfy a system of one-dimensional equations

$$
0=\Psi(x, \alpha) \equiv\left(\begin{array}{c}
\Psi^{1}(x, \alpha) \\
\vdots \\
\Psi^{n}(x, \alpha)
\end{array}\right) .
$$

Equations having the form (1) arise as first-order conditions in microeconomic

\footnotetext{
*This work was partially supported by the National Science Foundation under Grant ENG 77-28432 and the National Institutes of Health under Grant GM 23732-03.

fPlease address correspondence to Leigh Tesfatsion, Department of Economics, University of Southern California, Los Angeles, California 90007; (213) 743-4469.
} 
constrained optimization models, as defining characterizations for general equilibrium and macroeconomic policy models, and as steady-state solution characterizations for descriptive and optimal growth models.

If $\Psi: R^{n+1} \rightarrow R^{n}$ is continuously differentiable over a neighborhood of a point $\left(x^{0}, \alpha^{0}\right)$ in $R^{n+1}$ for which $\Psi\left(x^{0}, \alpha^{0}\right)=0$ and $\left|\Psi_{x}\left(x^{0}, \alpha^{0}\right)\right| \neq 0$, then the implicit-function theorem guarantees the existence of a continuously differentiable function $x: N\left(\alpha^{0}\right) \rightarrow R^{n}$ over some open neighborhood $N\left(\alpha^{0}\right)$ of $\alpha^{0}$ in $R$ such that

$$
\frac{d x}{d \alpha}\left(\alpha^{0}\right)=-\Psi_{x}\left(x\left(\alpha^{0}\right), \alpha^{0}\right)^{-1} \Psi_{\alpha}\left(x\left(\alpha^{0}\right), \alpha^{0}\right)
$$

Equation (2) is the fundamental relation underlying almost all comparative static studies in economics. The expression $d x\left(\alpha^{0}\right) / d \alpha$ is statically viewed as the formal solution $z$ to a system of linear algebraic equations $\Psi_{x} z=-\Psi_{\alpha}$. Given certain optimality or stability postulates which place sign restrictions on the Jacobian matrix $\Psi_{x}$, the solution $z$ often yields local qualitative information about system sensitivity to parameter variations at the point $\alpha^{0}$. However, it is generally unrealistic to attempt to actually solve for $z$ via (2) for systems of dimension $n$ greater than three, since explicit representation for $\Psi_{x}$ is then quite difficult.

The present paper views Equation (2) in a different light, as a differential system that can be integrated from initial conditions to yield the solution trajectory for $x$ as a function of $\alpha$. As will be clarified below, this view of Equation (2) opens up possibilities for the routine comparative static analysis of complex parametrized economic systems, either locally (at parameter points) or nonlocally (over parameter intervals of interest).

As it stands, Equation (2) is an analytically incomplete differential system in the sense that a closed-form representation for the inverse of the Jacobian matrix $\Psi_{x}(x, \alpha)$ as a function of $x$ and $\alpha$ is often not feasible. ${ }^{1} \mathrm{~A}$ recent paper [4] supplements Equation (2) with differential equations for the adjoint $A(\alpha)$ and determinant $\delta(\alpha)$ of $J(\alpha) \equiv \Psi_{x}(x(\alpha), \alpha)$. Recalling that $J(\alpha)^{-1}=$ $A(\alpha) / \delta(\alpha)$, an analytically complete differential system is thus obtained which generates joint solution trajectories for $x(\alpha), A(\alpha), \delta(\alpha)$.

Specifically, letting $\operatorname{Tr}(\cdot), \operatorname{Adj}(\cdot)$, and $|\cdot|$ denote the trace, adjoint, and determinant, respectively, the system takes the form of $n+n^{2}+1$ differential

\footnotetext{
${ }^{1}$ Numerical integration of Equation (2) would then require the supplementary solution of a system of linear algebraic equations at each step in the integration process, a computationally undesirable procedure. See [2] and [3].
} 
equations

$$
\begin{aligned}
& \frac{d x}{d \alpha}(\alpha)=-\frac{A(\alpha) \Psi_{\alpha}(x(\alpha), \alpha)}{\delta(\alpha)} \\
& \frac{d A}{d \alpha}(\alpha)=\frac{A(\alpha) \operatorname{Tr}(A(\alpha) B(\alpha))-A(\alpha) B(\alpha) A(\alpha)}{\delta(\alpha)} \\
& \frac{d \delta}{d \alpha}(\alpha)=\operatorname{Tr}(A(\alpha) B(\alpha)),
\end{aligned}
$$

subject to the initial conditions

$$
\begin{aligned}
& x\left(\alpha^{0}\right)=x^{0} \\
& A\left(\alpha^{0}\right)=\operatorname{Adj}\left(J\left(\alpha^{0}\right)\right), \\
& \delta\left(\alpha^{0}\right)=\left|J\left(\alpha^{0}\right)\right| \neq 0 .
\end{aligned}
$$

The $i j$ th component $b_{i j}(\alpha)$ of the $n \times n$ matrix $B(\alpha) \equiv d J(\alpha) / d \alpha$ is given by

$$
b_{i j}(\alpha) \equiv \sum_{m=1}^{n} \frac{\partial^{2} \Psi^{i}}{\partial x_{i} \partial x_{m}}(x(\alpha), \alpha) \frac{\partial x_{m}}{\partial \alpha}(\alpha)+\frac{\partial^{2} \Psi^{i}}{\partial x_{i} \partial \alpha}(x(\alpha), \alpha)
$$

As a theoretical tool, this new set of equations (3) extends the traditional use of (2) by allowing qualitative sensitivity analysis over parameter intervals. (See [4].)

A FORTRAN program COMSTAT with user instructions is now available upon request for the numerical integration of (3) over arbitrary parameter intervals $\left[\alpha^{0}, \alpha^{1}\right]$ for any $n \geqslant 1$. The only input requirements are the function $\Psi(\cdot)$ and the initial conditions (3d), (3e), (3f). (As will be clarified in Section 2, this initialization can generally be rendered routine by a simple two-stage application of comstat. ${ }^{2}$ ) The program automatically evaluates all needed first- and second-order partial derivatives of $\Psi(\cdot)$ using a generalized Wengert method [6]. The user is thus freed from ever having to analytically form the partial derivatives of $\Psi(\cdot)$. The numerical integration of the differential-equation system (3) is accomplished by a fourth-order Runge-Kutta start with an Adams-Moulton continuation.

\footnotetext{
${ }^{2}$ Since the initialization of (3) at $\alpha^{0}$ is equivalent to solving (2), routine initialization of (3) is equivalent to routine local comparative static analysis at $\alpha^{0}$.
} 
From a computational viewpoint, COMSTAT has two key advantages: It exploits the tremendous capability of present-day computers to solve, with great speed and accuracy, large systems of ordinary differential equations subject to initial conditions; and it totally avoids linear algebraic equations, a well-known source of trouble for computers.

Of course, examples can be constructed for which the comparative static analysis of the system (1) is difficult, or even virtually impossible. As with any computer program, the user must exercise reasonable care in assessing the results of the calculations. See, however, the numerically difficult optimal growth problem routinely handled by COMSTAT in Section 3 .

\section{PROGRAM INPUTS}

COMSTAT is applicable for all twice continuously differentiable functions $\Psi$ : $R^{n+1} \rightarrow R^{n}$ whose component functions $\Psi^{i}: R^{n+1} \rightarrow R, j=1, \ldots, n$, can be sequentially evaluated using special functions of one and two arbitrary variables $u$ and $(u, v)$. At present, COMSTAT's special-function library includes $\sin u, \cos u, J_{m}(u)$ (the $m$ th Bessel function), $\exp u, \log u, a u^{b}+c$ (for arbitrary constants $a, b, c), u+v, u-v, u v, u / v$, and $u^{v}$. Additional special-function subroutines can be added by the user.

The user must provide stylized instructions for the sequential evaluation of each component function $\Psi^{i}$ in a subroutine Fun. For example, consider the seemingly complicated function

$$
\Psi^{1}\left(x_{1}, x_{2}, x_{3}, \alpha\right) \equiv 3\left\{\sin \left[\log \left(x_{1}+x_{2}+x_{3}\right)\right]+\alpha x_{1}\right\}^{2} .
$$

This function can be sequentially evaluated using COMSTAT's special functions. The needed instructions for evaluation of (4) would appear in FUN as follows:

$\begin{array}{ll}\operatorname{CALL~AOD~}(\mathrm{X} 1, \mathrm{X} 2, \mathrm{X}) & \left(x=x_{1}+x_{2}\right), \\ \operatorname{CALL~ADD~}(\mathrm{X} 3, \mathrm{X}, \mathrm{W}) & \left(w=x_{3}+x\right), \\ \operatorname{CALL~LOG~}(\mathrm{W}, \mathrm{Z}) & (z=\log w), \\ \operatorname{CALL~SIN~}(\mathrm{Z}, \mathrm{Y}) & (y=\sin z), \\ \operatorname{CALL~MUL~}(\mathrm{X} 1, \mathrm{ALPHA}, \mathrm{U}) & \left(u=\alpha x_{1}\right), \\ \operatorname{CALL~ADD~}(\mathrm{Y}, \mathrm{U}, \mathrm{V}) & (v=y+u), \\ \text { CALL POW }(3, \mathrm{~V}, 2, \mathrm{~S}) & \left(s=3 v^{2}\right) .\end{array}$


For any specified values for $x_{1}, x_{2}, x_{3}, \alpha$, the subroutine (5) thus evaluates $s=\Psi^{1}\left(x_{1}, x_{2}, x_{3}, \alpha\right)$.

What the user need not know is that all of the first- and second-order partial derivatives of $\Psi^{1}$ required for the subsequent comparative static analysis of $\Psi(x, \alpha)=0$ are also evaluated and stored, together with the function value $\Psi^{1}(x, \alpha)$, whenever FUN is called. This is because all of the COMSTAT special-function subroutines called by FUN are calculus subroutines. They automatically compute the first and second partial derivatives of whatever special-function expression they are asked to evaluate, without the user having to have first formed the derivatives analytically. The idea of using calculus subroutines is originally due to Wengert [6]. See also Bellman et al. [1].

The only remaining input required of the user, apart from the integration step size, is that he initialize the system (3a), (3b), (3c) by providing initial conditions (3d), (3e), (3f) at some parameter value $\alpha^{0}$. In most cases initialization can be rendered routine by a simple two-stage application of cOMSTAT.

Specifically, suppose the basic problem of interest is the COMSTAT analysis of the system (1) starting at $\alpha^{0}$. If initialization of (3) at $\alpha^{0}$ is difficult, the user can first consider the modified system

$$
\Psi^{*}(x, \beta) \equiv \beta \Psi\left(x, \alpha^{0}\right)+[1-\beta] I x_{x}=0,
$$

where $I$ is the $n \times n$ identity matrix. Note that $\Psi^{*}(x, 1)=\Psi\left(x, \alpha^{0}\right)$. Initial conditions are easily provided for (7) starting at $\beta^{\prime}=0$; namely,

$$
\begin{aligned}
& x^{*}(0)=0, \\
& A^{*}(0)=I, \\
& \delta^{*}(0)=1 .
\end{aligned}
$$

In principle, сомSTAт can be used to integrate the differential system (3) for $\Psi^{*}(\cdot)$ from $\beta^{\prime}=0$ to $\beta^{\prime \prime}=1$. (Singular values $\beta$ where the determinant $\delta^{*}(\beta)$ is zero can generally be avoided by detouring into the complex plane.) The final trajectory values $x^{*}(1), A^{*}(1), \delta^{*}(1)$ then provide a set of initial conditions for the COMSTAT analysis of the original system (1) starting at $\alpha^{0}$, provided that $\delta^{*}(1) \neq 0$.

\section{OPTIMAL GROWTH EXAMPLE}

A standard one-sector optimal growth model will be used to illustrate COMSTAT. (See [5, pp. 444-450].) 


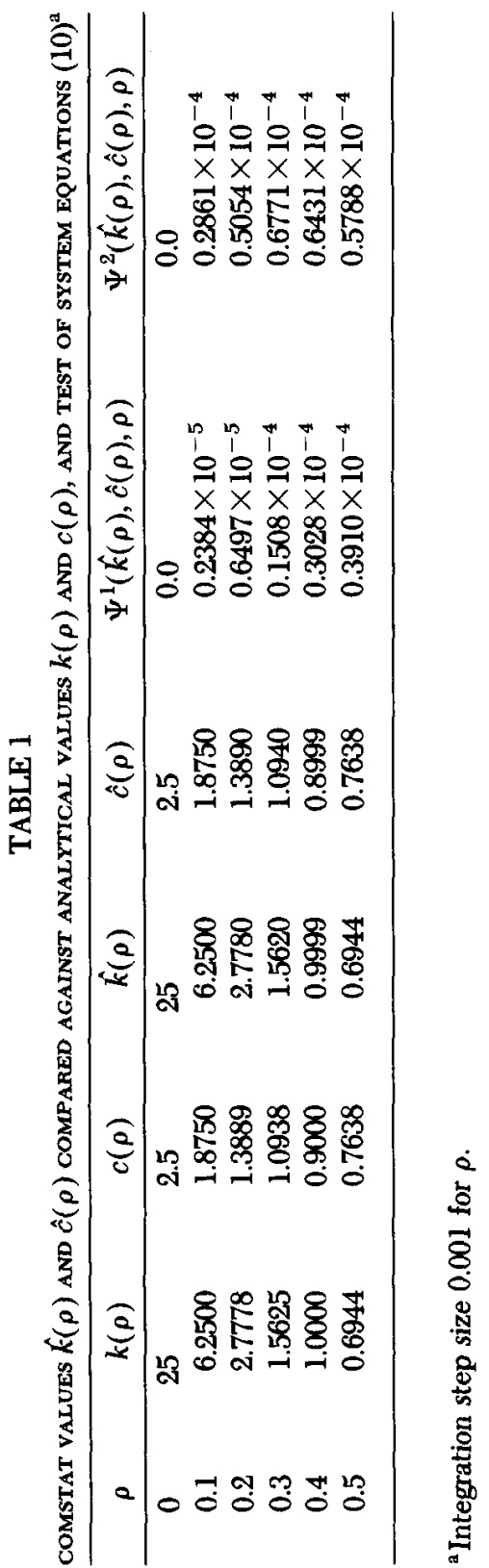


Consider the problem of selecting a per-capita capital trajectory $\mathbf{k}$ and a per-capita consumption trajectory $\mathrm{c}$ to maximize the discounted expected utility

$$
\int_{0}^{T} u(c(t)) e^{-\rho t} d t
$$

subject to the technological feasibility conditions

$$
\begin{aligned}
\dot{k}(t)+\lambda k(t)+c(t) & =f(k(t)), \quad 0 \leqslant t \leqslant T, \\
k(0) & =k^{0}>0,
\end{aligned}
$$

where $\lambda \equiv \delta+g$ is the sum of a capital depreciation rate $\delta>0$ and labor growth rate $g>0, f: R \rightarrow R$ is a twice continuously differentiable production function satisfying $f^{\prime \prime}<0$, and $u: R \rightarrow R$ is a twice continuously differentiable utility function satisfying $u^{\prime}>0$ and $u^{\prime \prime}<0$. Then necessary and sufficient conditions for a pair of values $(\bar{k}, \bar{c})$ to yield a steady-state solution for (9) are that $(\bar{k}, \bar{c})$ satisfy

$$
\begin{aligned}
& 0=f^{\prime}(k)-\lambda-\rho \equiv \Psi^{1}(k, c, \rho), \\
& 0=f(k)-\lambda k-c \equiv \Psi^{2}(k, c, \rho) .
\end{aligned}
$$

COMSTAT trajectory values were obtained for $\bar{k}$ and $\bar{c}$ as functions $\hat{k}(\rho)$ and $\hat{c}(\rho)$ of the time preference parameter $\rho$ over $[0,0.5]$ using the parameter specifications

$$
f(k) \equiv k^{1 / 2}, \quad \lambda \equiv 0.1, \quad \rho^{0}=0 .
$$

Table 1 illustrates the accuracy of COMSTAT by displaying how well the numerically generated values $\hat{k}(\rho)$ and $\hat{c}(\rho)$ approximate the analytically derived values $k(\rho)$ and $c(\rho)$ and satisfy the system equations (10). This optimal growth example is a stringent test of COMSTAT, for the derivative of $k(\rho)$ near $\rho=0$ is on the order of $-10^{2}$, whereas the derivative of $k(\rho)$ near $\rho=0.5$ is on the order of $-10^{\circ}$.

\section{REFERENCES}

1 R. Bellman, H. Kagiwada, and R. Kalaba, Wengert's numerical method for partial derivatives, orbit determination, and quasilinearization, Comm. ACM 8:231-232 (1965). 
2 D. Davidenko, Ob novom metode čislennogo reshenija sistem nelineinyh uravnenii, Dokl. Akad. Nauk SSSR 87:601-602 (1953).

3 R. Kalaba, E. Zagustin, W. Holbrow, and R. Huss, A modification of Davidenko's method for nonlinear systems, Comput. Math. Appl. 3:315-319 (1977).

4 R. Kalaba and L. Tesfatsion, Complete comparative static differential equations, Nonlinear Anal. 5:821-833 (1981).

5 Akira Takayama, Mathematical Economics, Dryden, Hinsdale, Illinois, 1974.

6 R. Wengert, A simple automatic derivative evaluation program, Comm. ACM 7:463-464 (1964). 